\title{
Perfil Epidemiológico dos Pacientes em Tratamento na Unidade de Fototerapia do Serviço Dermatologia de um Hospital Universitário
}

\author{
Jessica Lana Conceição e Silva Baka', Norami de Moura Barros², Allen de Souza Pessoa ${ }^{3}$, Luna Azulay-Abulafia ${ }^{4}$ \\ 'Pós-graduação em andamento em Dermatologia - Hospital Universitário Pedro Ernesto da Universidade do Estado do Rio de \\ Janeiro - Departamento de Dermatologia do Hospital Universitário Pedro Ernesto da Universidade do Estado do Rio de Janeiro, Rio \\ de Janeiro, Brasil. \\ ${ }^{2}$ Residência Médica em Dermatologia - Hospital Universitário Pedro Ernesto, Universidade do Estado do Rio de Janeiro. - Departamento \\ de Dermatologia, Hospital Universitário Pedro Ernesto, Universidade do Estado do Rio de Janeiro, Rio de Janeiro, Brasil. \\ ${ }^{3}$ Residência Médica em Dermatologia - Hospital Universitário Pedro Ernesto, Universidade do Estado do Rio de Janeiro - \\ Departamento de Dermatologia do Hospital Universitário Pedro Ernesto da Universidade do Estado do Rio de Janeiro, Rio de \\ Janeiro, Brasil \\ ${ }^{4}$ Doutora e Mestre em Dermatologia Universidade Federal do Rio de Janeiro, Professora Associada do Departamento de \\ Dermatologia HUPE - Universidade do Estado do Rio de Janeiro, Professora do Instituto de Dermatologia Prof. RDAzulay - Santa \\ Casa - RJ - Departamento de Dermatologia do Hospital Universitário Pedro Ernesto - Universidade do Estado do Rio de Janeiro, Rio \\ de Janeiro, Brasil
}

RESUMO - Introdução: A fototerapia é uma modalidade terapêutica empregada para tratamento de várias dermatoses. Consiste em exposição à radiação não ionizante, classificada em UVA ou UVB segundo seu comprimento de onda. Material e Métodos: Efectuámos um estudo observacional, transversal e descritivo no setor de fototerapia do Serviço de Dermatologia de um hospital universitário com o objectivo de avaliar os dados epidemiológicos e o grau de satisfação dos doentes. Resultados: Foram incluídos 51 indivíduos, 69\% eram do sexo feminino com uma idade média de 49 anos. As dermatoses em tratamento mais prevalentes durante a pesquisa foram: o vitiligo, com 62,5\% dos pacientes, e em segundo lugar a micose fungoide, com 13,73\%. Em relação ao nível de satisfação com o tratamento, mais de $90 \%$ se declararam satisfeitos/muito satisfeitos. Discussão: A prevalência de mulheres e fototipos mais baixos foi concordante com a literatura. A predominância de idosos nesse estudo corroborada com outros trabalhos e pode ser justificada pela presença de comorbidades, e assim contraindicações aos tratamentos convencionais além de maior disponibilidade de tempo do paciente. Em alguns casos a dermatose mais prevalente foi a psoríase, diferindo do nosso estudo. A segunda dermatose mais prevalente em nosso estudo foi a micose fungoide, o que difere de outros estudos nos quais a segunda dermatose mais prevalente foi a psoríase ou o vitiligo. Conclusão: Estudos como este são necessários para o planejamento tanto dos serviços que já dispõem dessa modalidade terapêutica quanto das unidades que desejam implantá-la. Nossos resultados não são integralmente convergentes com os da literatura publicada já que atualmente existem novas opções para o tratamento da psoríase.

PALAVRAS-CHAVE - Brasil; Fototerapia/epidemiologia; Fototerapia/estatística e dados numéricos.

\section{Epidemiological Profile of Patients Undergoing Treatment in the Phototherapy Unit of the Dermatology Service of a University}

ABSTRACT - Introduction: Phototherapy is a therapeutic modality used to treat various dermatoses. It consists of exposure to non-ionizing radiation, classified as UVA or UVB according to its wavelength. Material and Methods: This is an observational, cross-sectional and descriptive study carried out in the phototherapy sector of the Dermatology Service of a university hospital

Correspondência: Jessica Lana Conceição Boulevard 28 de Setembro, 77 - Vila Isabel Rio de Janeiro - RJ, 20551-030, Brasil E-mail: jessicabaka21@gmail.com

DOI: https://dx.doi.org/10.29021/spdv.78.3.1240
Recebido/Received 2020/06/23

(c) Autor (es) (ou seu (s) empregador (es)) 2020 Revista SPDV. Reutilização permitida de acordo com CC BY-NC. Nenhuma reutilização comercial.

(c) Author(s) (or their employer(s)) 2020 SPDV Journal. Re-use permitted under CC BY-NC. No commercial re-use. 


\section{Artigo Original}

with the objective of characterizing the population under treatment and their degree of satisfaction. Results: Fifty-one individuals were included of whom $69 \%$ were female, with a mean age of 49 years. The most prevalent dermatoses under treatment during the research were: vitiligo (62.5\%), and mycosis fungoides (13.73\%). Regarding the level of satisfaction with treatment, more than $90 \%$ declared themselves satisfied/very satisfied. Discussion: The prevalence of women and lower phototypes was consistent with the literature. The predominance of the elderly in this study corroborated by other studies may be justified by the presence of comorbidities, and contraindication to conventional treatments beside more time available for the treatment. In some cases, the most prevalent dermatosis was psoriasis, differing from our study. The second most prevalent dermatosis in our study was mycosis fungoides, which also differs from the studies found that described psoriasis or vitiligo as the second most prevalent dermatosis. Conclusion: Studies such as this one are necessary for the planning both the departments that already have this therapeutic modality and those that wish to implement it. Our results are not fully convergent with those of the literature as nowadays there are new options for the treatment of psoriasis.

KEYWORDS - Brazil; Phototherapy/epidemiology; Phototherapy/statistics and numerical data.

\section{INTRODUÇÃO}

A fototerapia é uma modalidade terapêutica empregada para tratamento de várias dermatoses. Essa prática existe desde 2000 AC. quando a helioterapia foi usada para tratar algumas doenças no Egito, Grécia e Índia, como o vitiligo. ${ }^{1,2}$ Consiste em exposição à radiação ultravioleta (UV)A ou UVB, no primeiro caso após administração de substância fotossensibilizante oral (psoraleno) - PUVA. ${ }^{1}$

Psoríase, vitiligo, linfoma cutâneo de células $T$, parapsoríase, dermatite atópica e outras dermatoses de caráter crônico podem ser tratadas com resultados satisfatórios. ${ }^{3-13}$ Para melhores resultados, pode ser associada a outros medicamentos sistêmicos, como os retinóides, especialmente no caso de psoríase, e menos frequentemente ao metotrexato ou ciclosporina, visando controle das dermatoses com doses menores desses medicamentos. ${ }^{3,7}$

São limitações da fototerapia a disponibilidade do equipamento, adesão do paciente, e considerações clínicas, como a dose cumulativa total dos raios UV e suas consequências. $^{3} \mathrm{O}$ efeito colateral agudo mais comum da terapia com UVB ou PUVA é a "queimadura solar" que ocorre cerca de 24 horas após o tratamento, ${ }^{8,9}$ mas pode também ocorrer uma erupção polimórfica à luz ou fotossensibilidade induzida por medicamentos. Os efeitos colaterais crônicos incluem lentigos, fotoenvelhecimento, queratoses actínicas e câncer de pele. ${ }^{8,9}$

Apesar do uso da fototerapia em diversas dermatoses e em vários centros pelo mundo, são poucos os dados consistentes sobre a população que utiliza esta terapêutica, ${ }^{10-12}$ sendo importantes estudos semelhantes para o planejamento de unidades que desejam implantar este método, além de esclarecer o tipo de paciente a selecionar e as dermatoses mais comumente tratadas. ${ }^{11,12}$

No departamento de Dermatologia do Hospital Universitário Pedro Ernesto do Rio de Janeiro, onde se utiliza fototerapia com UVA e UVB pelo há mais de 20 anos, este estudo foi realizado entre dezembro de 2018 e fevereiro de 2019 com a finalidade de caracterizar a população beneficiada com a fototerapia e o seu grau de satisfação.

\section{MATERIAL E METÓDOS}

Trata-se de um estudo observacional, transversal e descritivo realizado no setor de fototerapia do Serviço de Dermatologia de um hospital universitário de alta complexidade. Dispomos de dois equipamentos de radiação UV, uma cabine com 42 lâmpadas de 100W UVA (340 a 400 nm) e outra com 6 lâmpadas UVB narrow band (banda estreita) de $100 \mathrm{~W}(311$ a $312 \mathrm{~nm})$.

A colheita de dados foi realizada nos 3 meses entre dezembro de 2018 e fevereiro de 2019 entrevistando os pacientes em tratamento naquele período. Os dados foram obtidos através do preenchimento de um questionário aplicado por um dos pesquisadores. Nesse questionário os pacientes forneceram informações pessoais como idade, gênero, fototipo, diagnóstico, tempo para chegar ao hospital da sua casa, quantas sessões semanais, eventos adversos, comorbidades, se em algum momento este tratamento prejudicou atividades laborativas, qual a importância desse tratamento, como caracteriza a resposta, descrição de tratamentos prévios, beneficio secundário, satisfação com o tratamento, dentre outras.

Este estudo foi aprovado pelo Comitê de Ética em Pesquisa da instituição ( $n^{\circ} 17321219.3 .0000 .5259$ ). Os critérios de exclusão foram: menos de 10 sessões de fototerapia ou preenchimento incompleto do questionário aplicado.

\section{RESULTADOS}

Foram incluídos 51 indivíduos, $69 \%$ do sexo feminino, com uma idade média de 49 anos, variando de 10 a 81 anos, com predomínio de indivíduos na faixa etária dos 61 aos 75 anos (Fig. 1). O fototipo III foi o mais prevalente na amostra (55\%) (Fig. 2).

Em relação ao tipo de fototerapia, a maioria dos pacientes $(63 \%)$ é submetida à radiação ultravioleta $B$ de banda estreita (UVB-NB). No estudo $96,1 \%$ dos pacientes realizam as sessões de fototerapia no setor duas vezes por semana, raramente uma vez/semana. Em média, os pacientes estavam em tratamento entre três anos a cinco meses, a maior parte dos pacientes do estudo estão em tratamento mais de 2 anos.

As dermatoses em tratamento mais prevalentes durante a pesquisa foram: o vitiligo $(62,5 \%)$, a micose fungoide $(13,73 \%)$ e a psoríase $(7,48 \%)$. Outras dermatoses como 


\section{Artigo Original}

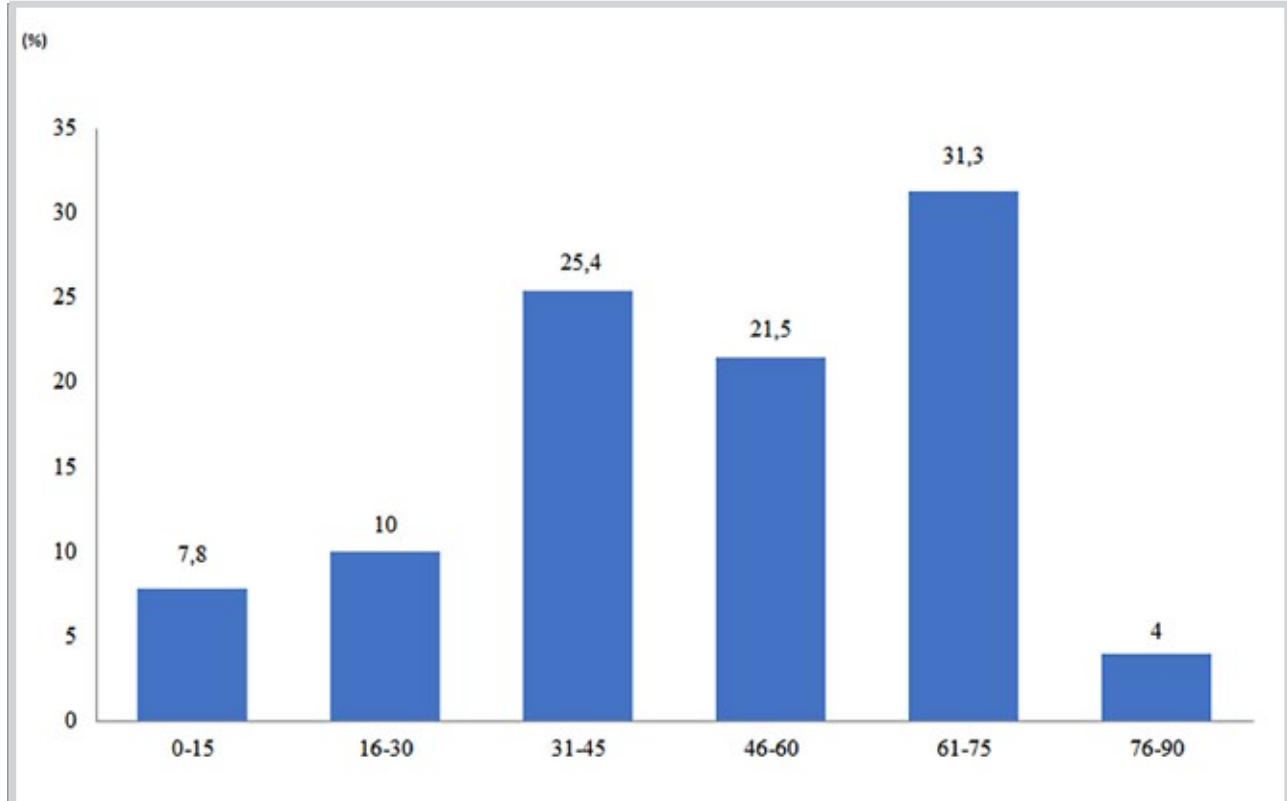

Figura 1 - Perfil etário dos pacientes.

parapsoríase, granuloma anular e esclerodermia tiveram menor prevalência (Fig. 3).

Em relação aos doentes com terapêuticas combinados nesse trabalho $78,43 \%$, faziam a fototerapia adjuvante a outras medicações como, por exemplo, tacrolimus ou metotrexato. O grau de melhora das dermatoses em tratamento com fototerapia foi de $98,0 \%$, sendo descrita uma melhora parcial ou significativa pelo paciente, isso corrobora com a satisfação do tratamento, que foi superior a 95\%.

Quanto aos efeitos colaterais da fototerapia, $78 \%$ dos pacientes afirmaram que durante $\circ$ tratamento houve um evento adverso, na sua grande maioria, a queimadura de primeiro grau $(90 \%)$.

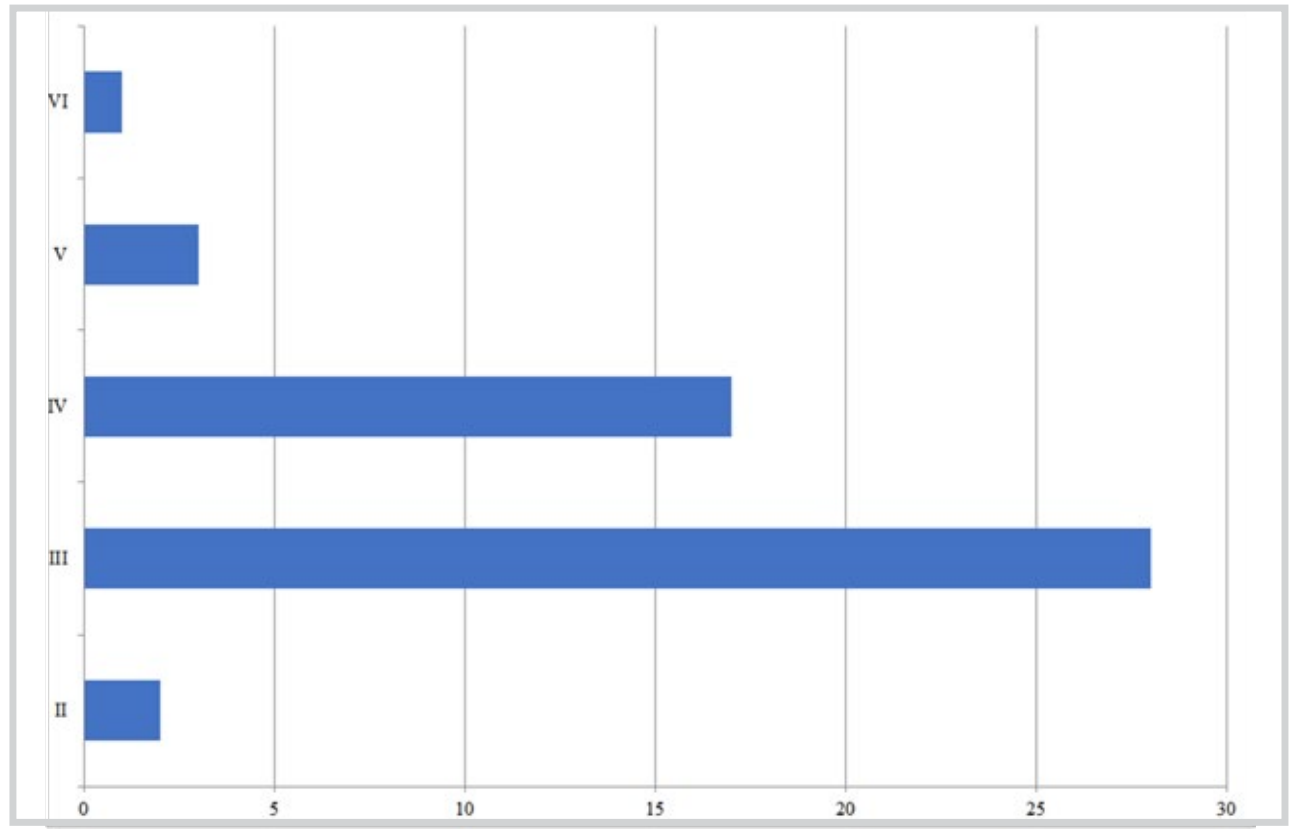

Figura 2 - Distribuição dos pacientes segundo o fototipo. 


\section{Artigo Original}

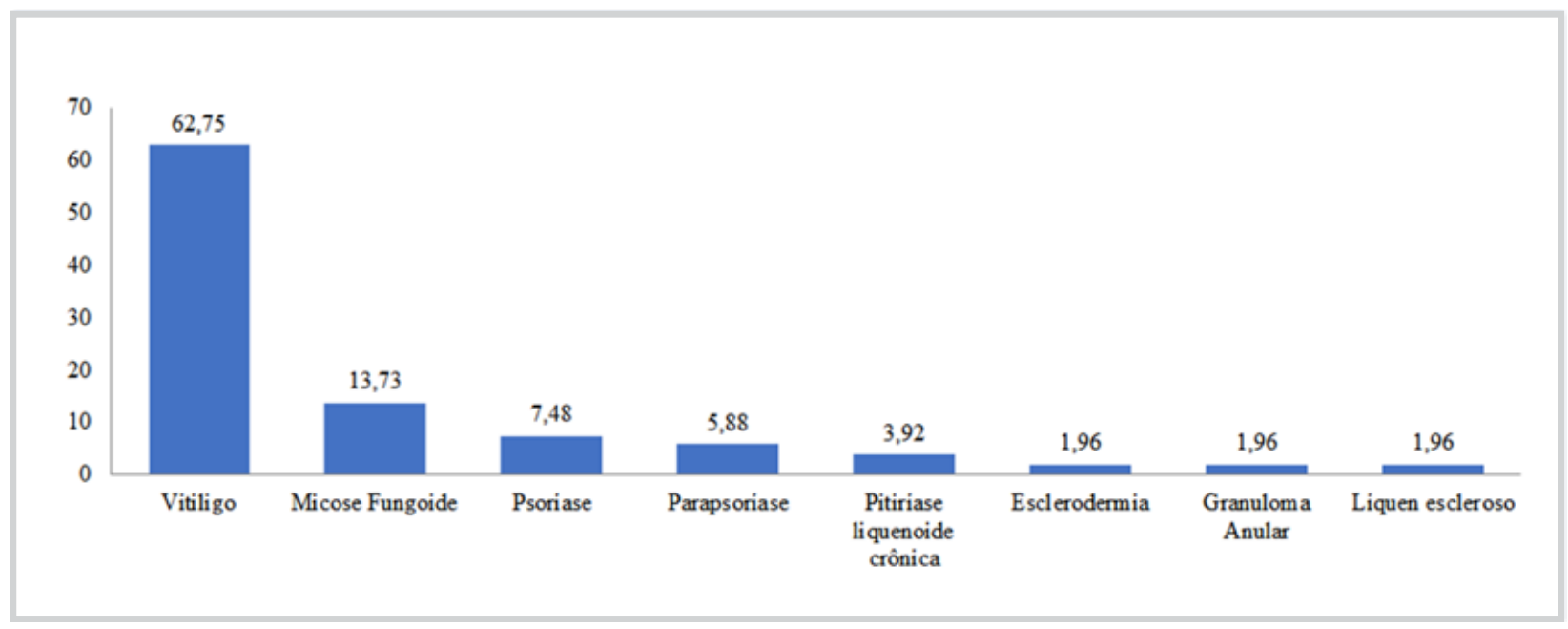

Figura 3 - Prevalência das dermatoses em tratamento com fototerapia.

Apenas $31 \%$ possuem um emprego formal, sendo que $88 \%$ afirmaram que não possuem restrições dentro do ambiente de trabalho devido ao tratamento. Quando questionados em relação ao nível de satisfação com o tratamento, $72 \%$ se declararam satisfeitos e $26 \%$ muito satisfeitos. Dentre os motivos pelos quais a fototerapia tem tal índice de satisfação além dos resultados terapêuticos objetivos, $22 \%$ dos pacientes mencionaram a socialização com os outros pacientes, constituindo praticamente um grupo terapêutico na sala de espera.

\section{DISCUSSÃO}

A prevalência de mulheres e fototipos mais baixos foi concordante com os achados de Casara et al e Ujihara et al, possívelmente, pela maior preocupação feminina com a saúde, estética e, ainda, perfil da população na região. ${ }^{11,12}$

A predominância de idosos nesse estudo é concordante com o trabalho de Ujihara et al e pode ser justificada pela presença de maior número de comorbidades nessa faixa etária, havendo assim mais contra-indicações aos tratamentos convencionais, além da maior disponibilidade de tempo do paciente, tornando a fototerapia uma opção terapêutica isolada ou adjuvante. ${ }^{11}$

Com relação a maior prevalência do vitiligo na amostra estudada, há concordância com os estudos Casara et al e Ujihara et al. ${ }^{11,12}$ A segunda dermatose mais prevalente em nosso estudo foi micose fungoide, o que difere dos estudos que avaliaram a prevalência das dermatoses tratadas com fototerapia e que apresentaram como segunda dermatose mais prevalente ou até a primeira, a psoríase. ${ }^{11,12,14-16}$ A justificativa para tal fato, é a disponibilidade de outras modalidades terapêuticas no tratamento da psoríase, permitindo que outras dermatoses beneficiem com a fototerapia, dada a limitação no número de vagas para o tratamento com fototerapia, no setor. ${ }^{11-13}$
Para submeter-se a fototerapia é necessária a disponibilidade de tempo e/ou condição social do paciente, mas em alguns casos essa é a única opção de tratamento seja por contraindicações a outras terapias coadjuvantes ou por limitadas opções terapêuticas. ${ }^{11,12}$

O efeito adverso mais prevalente, o número de sessões semanais, o uso da radiação UVB-NB e o uso combinado da fototerapia com outros medicamentos, são semelhantes com os dados da literatura. ${ }^{11,12}$

A fototerapia foi caracterizada como bem tolerada, segura e eficaz, semelhantes com os dados de Powell et al, sugerindo um provável aumento futuro na indicação e adesão a essa modalidade terapêutica. ${ }^{10}$

Embora o acesso à unidade de fototerapia constitua um importante fator limitante, o grau de satisfação e a socialização entre os pacientes, motivam o uso do método. Em relação ao grau de satisfação com o tratamento, nossos dados vão de acordo com a literatura, em que mais de $90 \%$ dos pacientes da amostra estavam satisfeitos com esta modalidade terapêutica. ${ }^{11}$

\section{CONCLUSÃO}

O uso da fototerapia como modalidade terapêutica na Dermatologia é uma opção na atualidade e fornece resultados satisfatórios e o grau de satisfação dos doentes é elevado. Este estudo permitiu descrever o perfil de um setor de fototerapia de um centro de dermatologia de referência, com dados importantes para o planejamento tanto de serviços que já dispõem dessa modalidade terapêutica quanto das unidades que desejam implantá-la.

\section{Agradecimentos}

Agradecemos ao grupo dos pacientes da fototerapia do Hospital Universitário Pedro Ernesto que gentilmente concordaram em participar da pesquisa. Sem eles, este trabalho não poderia ter sido realizado. 
Conflitos de interesse: Os autores declaram a inexistência de conflitos de interesse na realização do presente trabalho.

Fontes de financiamento: Não existiram fontes externas de financiamento para a realização deste artigo.

Confidencialidade dos dados: Os autores declaram ter seguido os protocolos da sua instituição acerca da publicação dos dados de doentes.

Protecção de pessoas e animais: Os autores declaram que os procedimentos seguidos estavam de acordo com os regulamentos estabelecidos pelos responsáveis da Comissão de Investigação Clínica e Ética e de acordo com a Declaração de Helsínquia da Associação Médica Mundial.

Proveniência e revisão por pares: Não comissionado; revisão externa por pares.

Conflicts of interest: The authors have no conflicts of interest to declare.

Financing support: This work has not received any contribution, grant or scholarship.

Confidentiality of data: The authors declare that they have followed the protocols of their work center on the publication of data from patients.

Protection of human and animal subjects: The authors declare that the procedures followed were in accordance with the regulations of the relevant clinical research ethics committee and with those of the Code of Ethics of the World Medical Association (Declaration of Helsinki).

Provenance and peer review: Not commissioned; externally peer reviewed

\section{ORCID}

Jessica Lana Conceição e Silva Baka

https://orcid.org/0000-0003-2995-2788

Norami de Moura Barros

https://orcid.org/0000-0001-9765-602X

Allen de Souza Pessoa

https://orcid.org/0000-0002-0608-8918

Luna Azulay-Abulafia

https://orcid.org/0000-0002-4698-2009

\section{REFERÊNCIAS}

1. Ledo E, Ledo A. Phototherapy, photochemotherapy, and photodynamic therapy: unapproved uses or indications. Clin Dermatol. 2000;18:77-86. doi:10.1016/S0738-081X(99)00096-6

2. Bolognia J, Jorizzo J, Schaffer J. Ultraviolet therapy. 3rd ed. New York: Elseiver; 2012

3. Duarte I, Buense R, Kobata C. Fototerapia. An Bras Dermatol. 2006;81:74 -82. doi:10.1590/S036505962006000100010
4. Duarte I, Cunha JAJ, Bedrikow RB, Lazzarini RR. Qual é o tipo de fototerapia mais comumente indicada no tratamento da psoríase? UVB banda estreita e PUVA:comportamento da prescrição. An Bras Dermatol. 2009;84:244-48. doi:10.1590/S036505962009000300005

5. Cestari TF, Pessato S, Corrêa GP. Fototerapia - aplicações clínicas. An Bras Dermatol. 2007;82:7-21. doi:10.1590/S0365-05962007000100002

6. Herzinger T, Berneburg M, Ghoreschi K, Gollnick H, Hölzle $E$, Hönigsmann $H$, et al. S1-Guidelines on UV phototherapy and photochemotherapy. J Dtsch Dermatol Ges. 2016;14:853-76 doi:10.1111/ddg.12912

7. Lim HW, Silpa-archa N, Amadi U, Menter A, Van Voorhees AS, Lebwohl M. Phototherapy in dermatology: A call for action. J Am Acad Dermatol. 2015; 72:107880. doi:10.1016/i.jaad.2015.03.017

8. Vangipuram R, Feldman S. Ultraviolet phototherapy for cutaneous diseases: a concise review. Oral Dis. 2015; 22:253-9. doi: 10.1111 /odi.12366

9. Zandi S, Kalia S, Lui H. UVA1 phototherapy: a concise and practical review. Skin Therapy Lett. 2012;17:1-4.

10. Powell JB, Gach JE. Phototherapy in the elderly. Clin Exp Dermatol. 2015;40:605-10. .hdoi:10.1111/ced.12626

11. Ujihara JED, Ferreira FR, Mandelbaum SH. Phototherapy: experience from a reference service. An Bras Dermatol. 2017;92:740-50.

12. Casara C, Eidt L, Cunha V. Prevalence study of dermatoses referred to the phototherapy unit at the Dermatology Service of the Clinics Hospital of Porto Alegre, RS, Brazil. An. Bras. Dermatol. 2013;88:211-5. doi:10.1590/ S0365-05962013000200004

13. Walker $D$, Jacobe $H$. Phototherapy in the age of biologics. Semin Cutan Med Surg. 2011;30:190-8. doi: $10.1159 / 000491782$

14. Tan SY, Buzney E, Mostaghimi A. Trends in phototherapy utilization among Medicare beneficiaries in the United States, 2000 to 2015. J Am Acad Dermatol. 2018;79:672-9. doi:10.1016/i.jaad.2018.03.018

15. Huynh NT, Sullivan JR, Commens CA. Survey of phototherapy practice by dermatologists in Australia. Aust. J. Dermatol. 2002;43:179-85. doi:10.1046/i.14400960.2002.00591.x

16. Aubin F, Courtois J, Puzenat E, Nardin C, Castelain F, Pelletier F, et al. Phototherapy in France: Quantitative Data (2007-2016) From the National Health Insurance Register. J Eur Acad Dermatol Venereol. 2018;32:e224-e225. doi: $10.1111 /$ jdv. 14746 